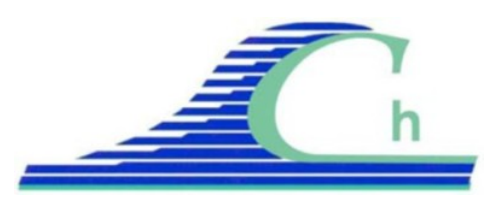

XII ${ }^{\text {èmes }}$ Journées Nationales Génie Côtier - Génie Civil

Cherbourg, $12-14$ juin 2012

DOI:10.5150/jngcgc.2012.032-KＣ Editions Paralia CFL

disponible en ligne - http://www.paralia.fr - available online

\title{
Dynamique sédimentaire des vasières intertidales de l'estuaire de la Loire
}

\section{Stéphane KERVELLA ${ }^{1}$, Aldo SOTTOLICHIO ${ }^{2}$, Eric MANEUX ${ }^{1}$, Christine BERTIER ${ }^{3}$, Jean-Baptiste LIBAUD ${ }^{4}$}

1. GEO-Transfert, Université de Bordeaux, UMR CNRS 5805, EPOC, Avenue des Facultés, 33405 Talence, France.

s.kervella@epoc.u-bordeauxl.fr

2. Université de Bordeaux, UMR CNRS 5805 - EPOC, Avenue des Facultés, 33405 Talence, France.

3. GIP Loire Estuaire, 22 rue la Tour d'Auvergne, 44200 Nantes, France.

4. HOCER, 23 Boulevard Einstein, 44323 Nantes cedex 3, France.

\section{Résumé :}

Au début des années 2000, un projet de réduction du bouchon vaseux par rectification de la morphologie de l'estuaire de la Loire a été confié au GIP Loire Estuaire (GIPLE). Les premières études ont conduit à privilégier la recréation de vasières intertidales sur la rive nord de l'estuaire. Afin de mieux comprendre l'influence des forçages hydrodynamiques et hydrologiques sur la dynamique sédimentaire de celles-ci, six Altus implantés sur deux vasières intertidales ont enregistré la hauteur des sédiments, la surface libre et les agitations de surface à haute fréquence pendant 17 mois.

\section{Mots-clés :}

Vasière intertidale - Hydrodynamique - Sédimentation - Vague - Estuaire de la Loire

\section{Introduction}

La géométrie de l'estuaire de la Loire a été fortement modifiée depuis 200 ans. Les aménagements successifs (chenalisation, comblement des bras secondaires, remblaiement des vasières, etc.) ont provoqué des modifications importantes de son hydrodynamique et des atteintes aux écosystèmes. Les réflexions menées depuis 2006 par le GIPLE sur ces problèmes ont conduits à l'élaboration d'un programme de restauration basé sur la rectification de la morphologie de l'estuaire. Un des principaux axes de ce programme consiste en la recréation de vasières afin de réduire le courant de flot, de piéger une partie des sédiments fins mais également de redévelopper le rôle de nourricerie pour les poissons, de zone d'alimentation pour les oiseaux et de renforcer la fonction épuratrice de l'estuaire. La sédimentation sur les vasières est complexe et dépend des forçages tidaux (CHRISTIES et al., 1999 ; PRITCHARD et al., 2002), du débit fluvial (DELOFFRE et al., 2005), de la position du bouchon vaseux (UNCLES et al., 1998), du vent (FAN et al., 2006), des bateaux (VERNEY et al., 2007) et également de l'activité biologique (ANDERSEN \& PEJRUP, 2002). Les dépôts de sédiments fins 
y sont temporaires, car soumis à des séquences de dépôt ou d'érosion de durée variable, avec des hauteurs pouvant aller de quelques millimètres par jour ou semaine à plusieurs dizaines de centimètres en quelques minutes (DELOFFRE et al., 2007). Avant d'entamer une dépoldérisation de certaines prairies, une étude a été lancée par le GIPLE entre 2008 et 2010, sur deux vasières de la partie aval de l'estuaire, afin de comprendre quelles sont les influences des forçages hydrodynamiques (marée, vagues) et hydrologiques (débit) sur leur sédimentation. Pour cela six altimètres ont été installés le long de deux transects cross-shore, mesurant à haute fréquence la hauteur de l'interface sédimentaire de la surface libre. Des prélèvements mensuels de l’interface sédimentaire ont également été réalisés.

\section{Site d'étude}

L’étude a été réalisée sur les vasières de "Mouton" et de "Pipy", localisées sur la rive droite de la partie aval de l'estuaire de la Loire (figure 1). La vasière de Mouton se situe à $12 \mathrm{~km}$ de l'embouchure de la Loire. Elle est caractérisée par une pente régulière (3,5\%) sur ses 250 premiers mètres à partir de la berge, sur laquelle 3 Altus ont été installés. La vasière de Pipy se situe à $17 \mathrm{~km}$ de l'embouchure de la Loire. Elle est caractérisée par une première pente raide (4\%) sur laquelle deux Altus ont été installés. Ensuite, la vasière présente un plat (pente de $0,5 \%$ sur une centaine de mètres) sur lequel a été positionné le dernier Altus. Les courants de marée peuvent, au-dessus de ces vasières, atteindre des vitesses supérieures à $0,8 \mathrm{~m} / \mathrm{s}$ en flot et $0,6 \mathrm{~m} / \mathrm{s}$ en jusant. Ces vasières sont proches du chenal de navigation.

\section{Méthodologie}

Six altimètres Altus (NKE) ont été déployés le long de deux lignes cross-shore (figure 1), entre décembre 2008 et avril 2010. Les Altus sont des échosondeurs qui enregistrent les variations verticales du fond $( \pm 0,2 \mathrm{~mm})$ et la hauteur d'eau ( $\pm 20 \mathrm{~mm})$. Sur chaque vasière, ils ont été positionnés à trois altitudes différentes (CM 96) : $4 \mathrm{~m}$ (Altus bas), 2,5 $\mathrm{m}$ (Altus milieu) et $1 \mathrm{~m}$ (Altus haut). Les Altus bas et milieu ont enregistré le signal à une fréquence de $2 \mathrm{~Hz}$ (mode "vague"). La hauteur significative des vagues $\left(\mathrm{H}_{\mathrm{s}}\right)$ leur période représentative $\left(T_{r}\right)$ et la contrainte de cisaillement générée par les vagues sur le fond $\left(\tau_{\mathrm{w}}\right)$ ont été calculées par la méthode spectrale de BASSOULET et al. (2010). Une correction du signal altimétrique (DELOFFRE et al., 2005) a été réalisée à partir des mesures de température et de salinité issues du réseau SYVEL (figure 1). Les cinq premiers centimètres de l'interface sédimentaire ont également été prélevés (analyse granulométrique par gravimétrie, norme NF X 31-107), afin de connaître la nature des sédiments et de pouvoir estimer la longueur de rugosité $\left(\mathrm{z}_{0}\right)$ entrant dans le calcul de $\tau_{\mathrm{w}}$. Dans le cadre de cette étude et d'après la nature des sédiments rencontrés, $\mathrm{z}_{0}=0,7 \mathrm{~mm}$, représentatif d'un fond sablo-vaseux (SOULSBY 1997). Il est à noter que des problèmes liés à la détérioration du matériel, de configuration, d'alimentation et 


\section{XII ${ }^{\text {èmes }}$ Journées Nationales Génie Côtier - Génie Civil \\ Cherbourg, 12-14 juin 2012}

d'envasement des Altus ont provoqué des lacunes parfois importantes dans les enregistrements, notamment sur les parties hautes des vasières (\% fonctionnement: Mouton haut $=21$, centre $=83$ et bas $=56$; Pipy haut $=42$, centre $=83$ et bas $=56$ ) .

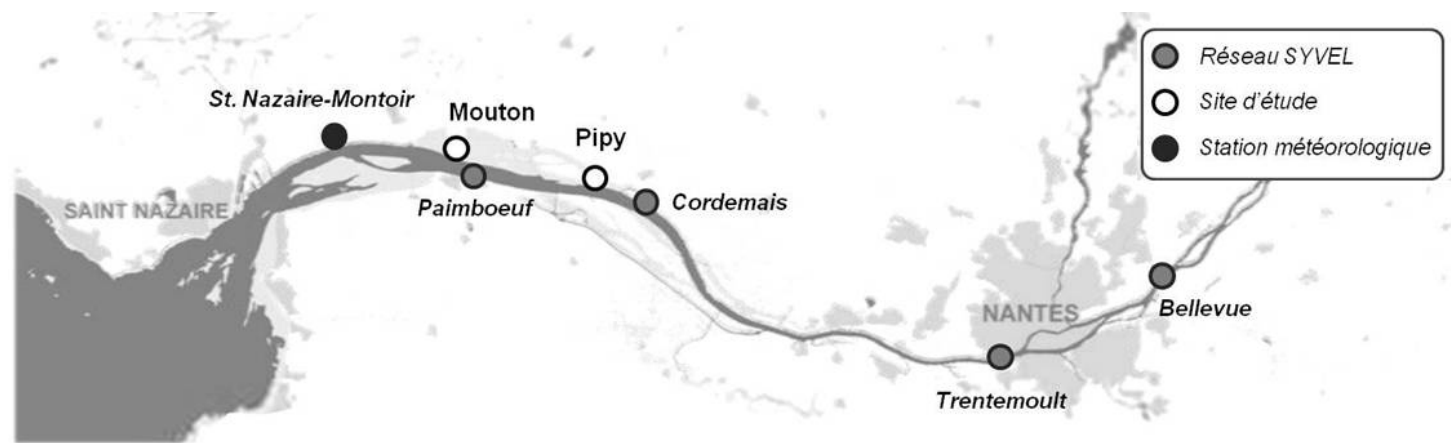

Figure 1. Position des sites d'étude dans l'estuaire de la Loire.

\section{Résultats}

4.1 Caractéristiques texturales des sédiments de surface

Les sédiments superficiels des parties hautes et centrales des vasières sont constitués de vases argilo-sableuses, tandis que ceux des parties basses sont des sables argileux (FLEMMING, 2000). Leur composition est relativement stable au cours de l'année, bien que des enrichissements en argile et en silt soient à signaler lors des premières crues.
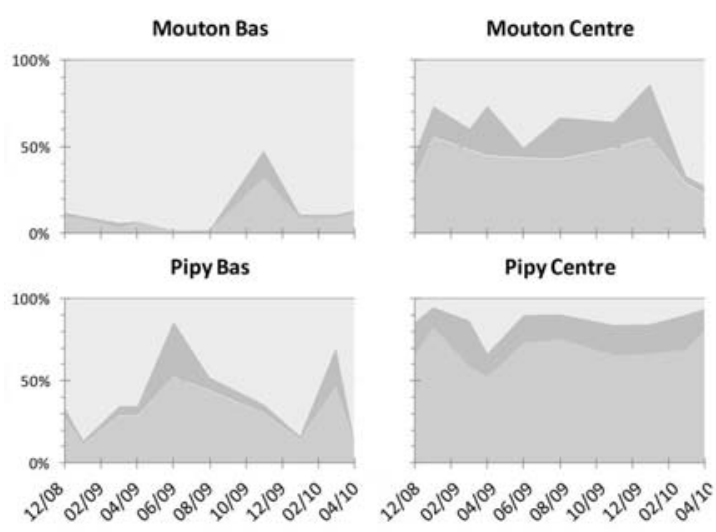

Pipy Centre

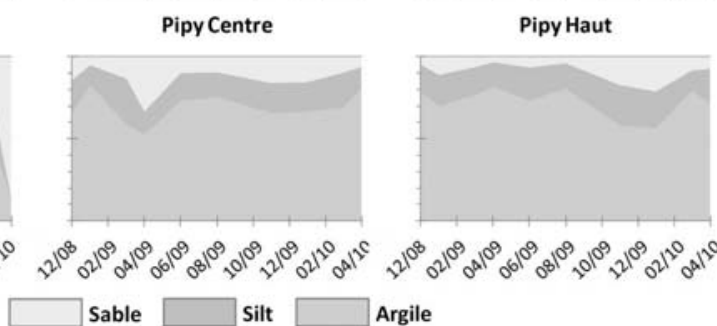

Figure 3. Variations des caractéristiques texturales des sédiments de surface des vasières de Mouton et de Pipy, entre décembre 2008 et avril 2010

\subsection{Réponse de l'interface sédimentaire à l'action des vagues}

Le plan d'eau au-dessus des vasières est la plupart du temps très calme (figure 3). Les tempêtes génèrent des houles longues qui se propagent depuis l'embouchure de l'estuaire jusque sur les vasières, où elles arrivent fortement atténuées (Hs=0,4 à $0,8 \mathrm{~m})$. 
Le développement des vagues dans l'estuaire est maximal lors des pleines mers. Elles génèrent des contraintes de frottement sur le fond importantes ( 1 à 3,5 N/m²), pouvant entrainer des érosions pluri-centimétriques. Ces érosions sont mal confirmées par les mesures Altus, mais elles ont été observées sur le terrain, 2 jours après la tempête Xynthia en mars 2010 (HOCER, 2010).

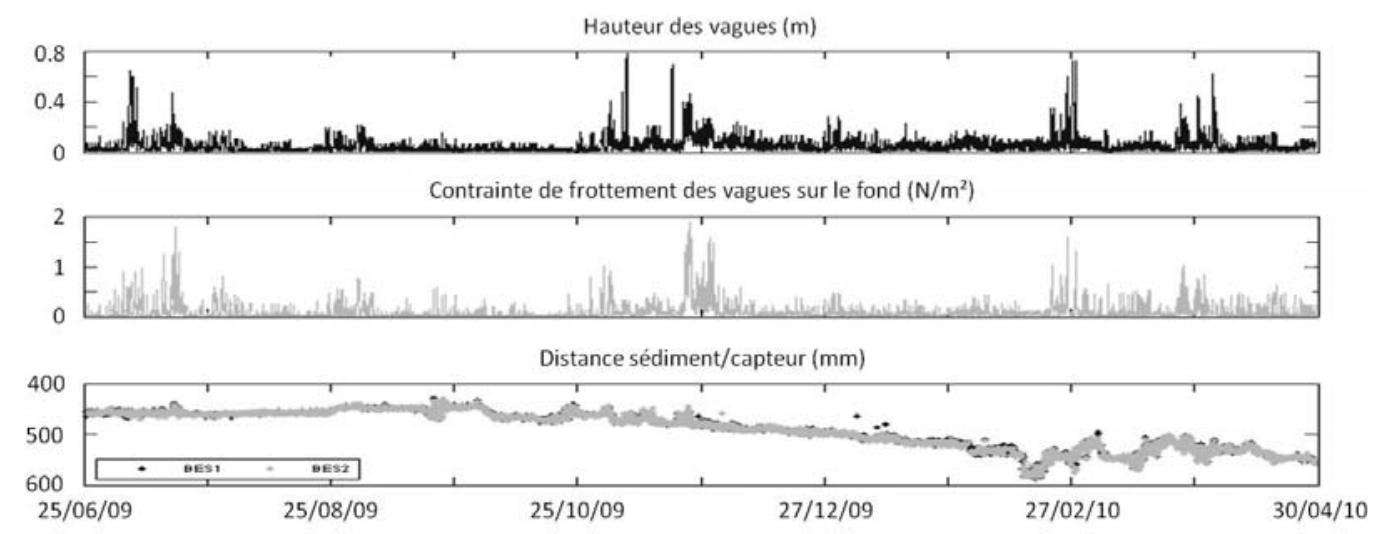

Figure 3. Enregistrement en continu des caractéristiques des vagues (Hs et $\tau w$ ) et la hauteur des sédiments sur le centre de la vasière de Mouton.

4.3 Influence de la marée et des débits fluviaux sur la stabilité des vasières

- Echelle annuelle - Influence des débits :

Entre le 15/12/08 et le 30/04/10, deux périodes de crues moyennes sont visibles. Les débits oscillent entre 600 et $1600 \mathrm{~m}^{3} / \mathrm{s}$, et présentent des pics supérieurs à $2000 \mathrm{~m}^{3} / \mathrm{s}$. En étiage, les débits sont très faibles $\left(<200 \mathrm{~m}^{3} / \mathrm{s}\right)$. Les variations altimétriques saisonnières sont similaires sur les deux vasières, et sont souvent corrélées au débit de la Loire (figure 4). En étiage, le centre et le haut des vasières est stable, tandis que les parties basses subissent de fortes variations, indépendantes du débit. Une forte érosion de vase peu consolidée $(30 \mathrm{~cm}$ ) a lieu sur le bas de Pipy, en étiage et viveseaux. Lors des premières crues, le dépôt est important $(>60 \mathrm{~cm})$ sur le bas des vasières, recouvrant totalement les Altus et entrainant une interruption dans l'acquisition des données. Ce dépôt n'est pas lié à une plus forte turbidité, mais plutôt à un déplacement amont-aval de la crème de vase sur le fond de l'estuaire. Ce dépôt est presque entièrement érodé quelques jours après. Les crues suivantes provoquent un dépôt continu sur le bas des vasières, alors que les parties centrales et hautes sont en érosion (0 à -10cm en 4 mois).

- Echelle pluri-mensuelle-Action des cycles de marée :

En étiage, en présence du bouchon vaseux, la hauteur de l'interface sédimentaire varie fortement, uniquement sur les points les plus bas des vasières (figure 5), en relation avec les cycles de marée (revif/déchet). En déchet, les particules en suspension décantent massivement, provoquant des dépôts de plusieurs dizaines de 


\section{XII ${ }^{\text {èmes }}$ Journées Nationales Génie Côtier - Génie Civil \\ Cherbourg, 12-14 juin 2012}

centimètres. Ces dépôts peuvent subir un tassement avant qu'ils ne soient érodés en période de revif. Les flux sédimentaires calculés sont de l'ordre de 3 à $9 \mathrm{~kg} / \mathrm{m}^{2} / \mathrm{j}$. En crue, le bouchon vaseux est positionné plus en aval, entre l'embouchure et la vasière de Mouton. Son influence n'est pas ressentie au niveau de la vasière de Pipy.

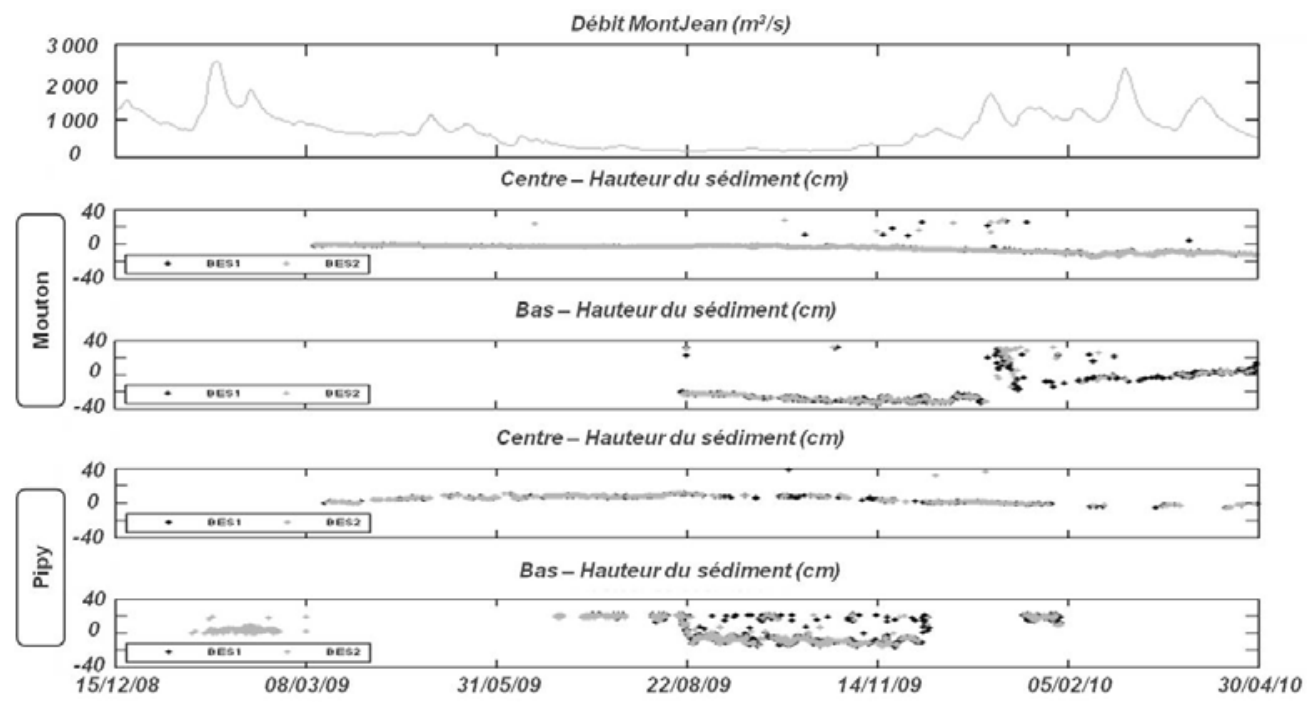

Figure 4. Variations altimétriques annuelles mesurées sur le bas et le centre des vasières de Mouton et de Pipy en fonction du débit de la Loire.
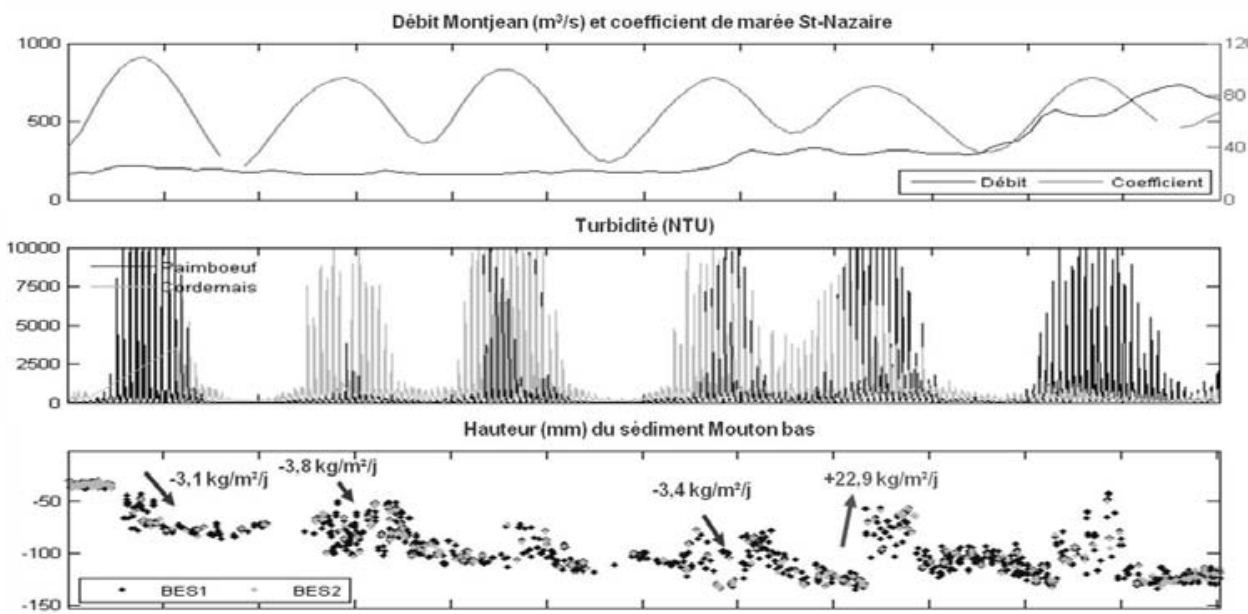

Hauteur ( $\mathrm{mm}$ ) du sédiment Pipy bas

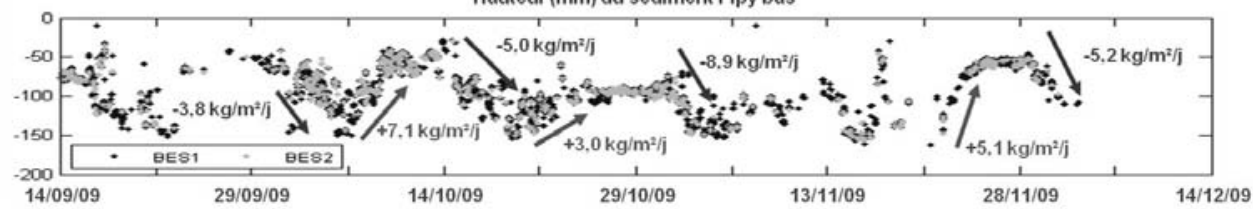

Figure 5. Hauteur du sédiment et estimation des flux sédimentaire en $\mathrm{kg} / \mathrm{m}^{2} / \mathrm{j}$ associés (Concentration des sédiments: $200 \mathrm{~g} / \mathrm{l}$ ) en fonction du débit journalier, de la turbidité à Paimboeuf et à Cordemais et des coefficients de marée. 
- Echelle semi-diurne - Action de la marée :

A l'échelle semi-diurne, l'interface sédimentaire varie de quelques mm à quelques $\mathrm{cm}$, en fonction de l'amplitude de la marée et de la présence ou non du bouchon vaseux (figure 6). La comparaison des deux vasières montre une dynamique plus importante sur la vasière de Pipy que sur la vasière de Mouton, avec des épaisseurs remaniées plus élevées, tout comme les flux sédimentaires associés (tableau 1). L’étude intra-vasière montre que les séquences de dépôt/érosion et les flux sédimentaires sont plus forts sur les parties basses des vasières. D'une manière générale, la présence du bouchon vaseux favorise les flux verticaux, ces derniers pouvant être amplifiés en période de vive-eau.

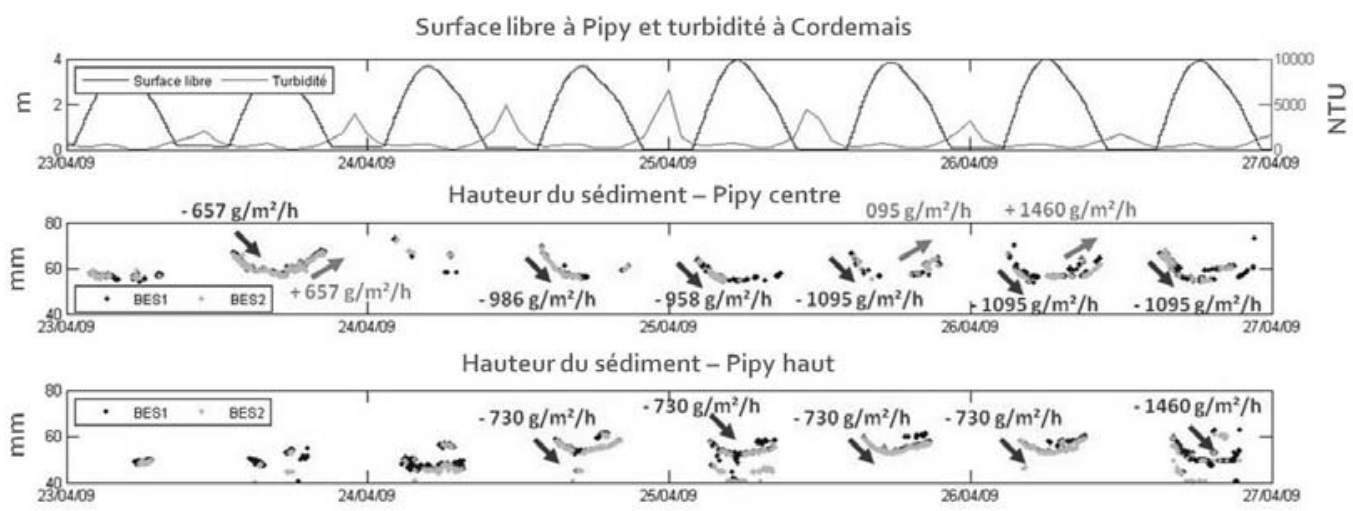

Figure 6. Variations altimétriques et flux sédimentaire horaire associé, en fonction de la hauteur d'eau à Pipy et de la turbidité enregistrée à la station Cordemais (SYVEL).

Tableau 1. Estimations des variations altimétriques horaires et des flux sédimentaires horaires associés, sur les vasières de Mouton et de Pipy, en fonction du régime hydraulique, de la présence du bouchon vaseux et du type de marée.

\begin{tabular}{|c|c|c|c|c|c|c|c|c|}
\hline \multirow{3}{*}{$\begin{array}{c}\text { Régime } \\
\text { hydraulique }\end{array}$} & \multirow{3}{*}{$\begin{array}{c}\text { Bouchon } \\
\text { vaseux }\end{array}$} & \multicolumn{6}{|c|}{$\begin{array}{l}\text { Hauteur moyenne des dépôts/érosion }(\mathrm{mm} / \mathrm{h}) \\
\text { Flux moyen d'érosion/dépôt }\left(\mathrm{g} / \mathrm{m}^{2} / \mathrm{h}\right)\end{array}$} & \\
\hline & & Type de & & Mouton & & & Pipy & \\
\hline & & Marée & Bas & Centre & Haut & Bas & Centre & Haut \\
\hline \multirow{3}{*}{ Etiage } & Oui & $V E$ & $15 / 2990$ & $7 / 1360$ & - & $26 / 5170$ & $5 / 990$ & - \\
\hline & \multirow{2}{*}{ Non } & $M E$ & $4 / 760$ & $3 / 590$ & - & $7 / 1370$ & $4 / 478$ & $5 / 950$ \\
\hline & & $V E$ & $4 / 840$ & $3 / 500$ & - & $2 / 410$ & - & - \\
\hline \multirow{3}{*}{ Crue } & Non & $V E$ & - & - & - & $37 / 7450$ & - & $4 / 720$ \\
\hline & \multirow{2}{*}{ Oui } & $V E$ & - & $29 / 5820$ & - & - & - & - \\
\hline & & $M E$ & $9 / 1760$ & $6 / 1200$ & - & - & - & - \\
\hline
\end{tabular}

\section{Discussion et conclusions}

Cette étude, basée sur la mesure continue par Altus, a permis de mieux comprendre les processus sédimentaires sur les vasières intertidales de la Loire. L'ensemble des 


\section{XII èmes Journées Nationales Génie Côtier-Génie Civil \\ Cherbourg, 12-14 juin 2012}

mécanismes d'érosion/dépôt (de la minute à l'année), ainsi que les rôles respectifs des forçages hydrodynamiques, ont été décrits et quantifiés.

Dans cette portion de l'estuaire, les tempêtes génèrent des vagues, qui sont la combinaison des houles du large et du clapot. Les contraintes de frottement sur le fond sont importantes (de 1 à $3,5 \mathrm{~N} / \mathrm{m}^{2}$ ), et sont comparables à celles calculées sur les vasières de la Seine (VERNEY, 2007), de la baie du Mont Saint-Michel et de la baie de Marennes-Oléron (KERVELLA, 2009; KERVELLA, 2010). L'impact sur la sédimentation est surtout vrai autour des étales de marée basse.

A l'échelle de la marée semi-diurne, l'interface sédimentaire des zones intertidales est remaniée en permanence. Les variations altimétriques sont faibles, mais leur amplitude varie en fonction du débit et des marées. A l'échelle pluri-mensuelle, la sédimentation est contrôlée par la position du bouchon vaseux (et donc par le débit) et par l'amplitude de la marée. En étiage, les variations altimétriques sont dues aux cycles des marées. En déchet, les matières en suspensions décantent sur les parties basses des vasières, sous la forme de vase fluide de plusieurs $\mathrm{cm}$ d'épaisseur, qui peut se tasser rapidement. En revif, l'augmentation de la vitesse des courants favorise l'érosion.

En crue, le bouchon vaseux descend dans la partie aval de l'estuaire. L'augmentation de la charge particulaire amplifie les échanges entre la colonne d'eau et le fond, notamment sur les parties basses des vasières. La proximité du chenal de navigation favorise l'accrétion continue. En crue, le renforcement de la vitesse de l'écoulement, semble limiter les dépôts dans les parties centrales et hautes, et favorise l'érosion des sédiments. Les premières crues remettent en suspension, ou font s'écouler sur le fond, des masses importantes de crème de vase stockées en amont en étiage, avec un effet de "vidange" des zones amont. Les sédiments en suspension vont alors se déposer en masse sur les parties basses des vasières et dans les chenaux (dépôts $>60 \mathrm{~cm}$ ). Ces dépôts, fluides, sont ensuite érodés et remis en suspension quelques jours plus tard. En crue, la variation de l'amplitude des marées ne semble pas modifier la sédimentation sur ces vasières.

Globalement, c'est la dynamique typiquement estuarienne qui contrôle la sédimentation. Les vagues sont des phénomènes épisodiques énergétiques ; même si leur action érosive n’a pas été enregistrée, elle semble efficace pour des faibles tranches d'eau.

\section{Références bibliographiques}

ANDERSEN T.J., PEJRUP M. (2002). Biological mediation of the settling velocity of bed material eroded from an intertidal mudflat, the Danish Wadden Sea. Estuar. Coast. Shelf S., 54, pp 737-745. doi:10.1006/ecss.2001.0856

BASSOULLET P., VERNEY R., KERVELLA Y., KERVELLA S., JESTIN H., VOINESON G. (2010). Utilisation d'un altimètre (ALTUS) destiné à la quantification des dépôt/érosion en domaine littoral pour l'étude des corrélations avec les caractéristiques de vagues et les interfaces de dépôt. Houille Blanche, (5), pp 81-86. doi:10.1051/lhb/2010058 
CHRISTIES M.C., DYER K.R., TURNER P. (1999). Sediment flux and bed-level measurements from a macrotidal mudflat. Estuar. Coast. Shelf S., 49, pp 667-688. doi:10.1006/ecss.1999.0525

DELOFFRE J., LAFITE R., LESUEUR P., LESOURD S., VERNEY R., GUEZENNEC L. (2005). Sedimentary processes on a fluvial estuarine mudflat: the macrotidal Seine example (France). Estuar. Coast. Shelf S., 64, 710-720. doi:10.1016/j.ecss.2005.04.004

DELOFFRE J., VERNEY R., LAFITE R., LESUEUR P., LESOURD S., CUNDY A. (2007). Sedimentation on intertidal mudflats in the lower part of macrotidal estuaries: Sedimentation rhythms and their preservation. Marine Geology, 241(1-4), pp 19-32. doi:10.1016/j.margeo.2007.02.011

FAN D., GUO Y., WANG P., SHI J.Z. (2006). Cross-shore variations in morphodynamic processes of an open-coast mudflat in the Changjiang delta, China: with an emphasis on storm impacts. Cont. Shelf Res., 26, pp 517-538. doi:10.1016/j.csr.2005.12.011

FLEMMING B.W. (2000). A revised textural classification of gravel-free muddy sediments on the basis of ternary diagrams. Continental Shelf Research, 20 (10-11), pp 1125-1137. doi: 10.1016/S0278-4343(00)00015-7

HOCER (2010). Mise en place des altimètres sur les vasières de la Loire: Bilan des données collectées. Rapport G.I.P. Loire Estuaire. Référence HCR/BT/10/GIPLE/RE.

KERVELLA S. (2009). Dynamique des sédiments fins et mixtes des zones intertidales de la baie de Marennes-Oléron. Thèse de Doctorat, Université de La Rochelle.

KERVELLA Y. (2010). Impact des installations ostréicoles sur l'hydrodynamique et la dynamique sédimentaire. Thèse de Doctorat, Université de Caen Basse-Normandie.

KERVELLA S., MANEUX E., SOTTOLICHIO A. (2011) Analyse des mesures enregistrées par 6 altimètres implantés sur des vasières de la Loire. Rapport GEOTransfert pour le G.I.P. Loire Estuaire

PRITCHARD D., HOGG A.J., ROBERTS R. (2002) Morphological modelling of intertidal mudflats: the role of cross-shore tidal currents. Cont. Shelf Res., 22, pp 18871895. doi:10.1016/S0278-4343(02)00044-4

SOULSBY R.L. (1997). Dynamics of marine sands. A manual for practical applications. Thomas Telford, London.

UNCLES R.J., EASTON A.E, GRIFFITHS M.L., HARRIS C.K., HOWLAND R.J.M., KING R.S., MORRIS A.W., PLUMMER D.H. (1998). Seasonality of the turbidity maximum in the Humber Estuary, UK, Marine Pollution Bulletin, 37 (3-7), pp 206-215. VERNEY R., DELOFFRE J, BRUN COTTAN J.-C., LAFITE R (2007). The effect of wave-induced turbulence on intertidal mudflats: Impact of boat traffic and wind. Continental Shelf Research, 27(5), pp 594-612. doi:10.1016/j.csr.2006.10.005 